\section{Orquiepididimitis brucelósica}

\section{Sr. Director:}

La brucelosis es una zoonosis endémica de algunos países en vías de desarrollo y zonas rurales de países desarrollados. Se transmite por el contacto con animales enfermos, el consumo de productos lácteos no pasteurizados y la inhalación de partículas infectadas $(1,2)$. La Brucella spp. puede afectar a múltiples órganos y tejidos, la orquiepididimitis es una manifestación poco frecuente descrita en el 2-20\% de las brucelosis sistémicas (2-5). Presentamos el caso de un varón joven, residente en un área urbana con baja incidencia de la enfermedad, que tenía el antecedente epidemiológico de un viaje a un área endémica de brucelosis y el consumo de productos lácteos no pasteurizados.

Varón de 37 años, sin antecedentes médicos de interés, que consulta por un cuadro de once días de evolución de fiebre termometrada de hasta $40,5^{\circ} \mathrm{C}$, de predominio matutino y vespertino, que en los últimos días se acompaña de molestias en el testículo derecho cuando realiza un esfuerzo físico. El mes anterior había realizado un viaje a Extremadura, donde consumió queso y leche de cabra sin pasteurizar. Al ingreso presentaba fiebre de $40,2{ }^{\circ} \mathrm{C}$ y taquicardia sinusal a 105 latidos por minuto, la tensión arterial era normal. En la exploración física sólo se objetivaba que el testículo derecho estaba aumentado de tamaño sin transiluminación, tumefacto, doloroso a la palpación y con zonas de fluctuación.

En las exploraciones complementarias realizadas destacaba: una analítica de sangre con 14.610 leucocitos $/ \mathrm{mm}^{3}, 77 \%$ neutrófilos, sin desviación izquierda y una VSG de $40 \mathrm{~mm}$ a la primera hora; una analítica de orina sin hallazgos patológicos y una ecografía testicular con un aumento inhomogéneo del tamaño del testículo derecho, con pequeñas zonas hipoecoicas y una mínima cantidad de líquido en la bolsa escrotal. El rosa de Bengala, el test Brucellacapt (1/5120) y el test de Coombs (1/1280) fueron positivos. En los hemocultivos se aisló Brucella spp. a los pocos días de haberlos realizado.

Realizó tratamiento con doxiciclina, $100 \mathrm{mg}$ cada 12 horas durante 6 semanas, asociando un gramo intramuscular de estreptomicina al día durante dos semanas. El paciente evolucionó de forma satisfactoria con desaparición de la sintomatología y normalización del tamaño testicular.

La afectación genitourinaria es infrecuente en la infección por Brucella spp, la orquiepididimitis generalmente es unilateral y no suele ocasionar atrofia o esterilidad $(2,5)$. Se distingue clínicamente de otras formas de orquiepididimitis por la presencia de fiebre ondulante, un inicio gradual con un curso prolongado, la presencia de menor inflamación local, la ausencia de sintomatología urinaria y el contacto con animales o el consumo de derivados lácteos no pasteurizados $(3,5,6)$.

La aproximación diagnóstica puede realizarse mediante las pruebas serológicas: rosa de Bengala, test de Coombs y test Brucellacapt. El diagnóstico definitivo se obtiene cuando se aísla la Brucella spp., generalmente en los hemocultivos $(2,3,5)$. La importancia de la ecografía reside más en su capacidad para descartar un absceso o un tumor, que en su utilidad para proporcionar el diagnóstico etiológico (5).

El tratamiento adecuado precisa de la combinación de dos antibióticos durante un periodo de tiempo prolongado, como mínimo seis semanas. La pauta más utilizada es la doxiciclina asociada a un aminoglucósido; se pueden emplear cotrimoxazol, doxiciclina o ciprofloxacino asociados a rifampicina obteniéndose similares resultados $(2,3,5,6)$. La forma necrotizante responde mal al tratamiento antibiótico y generalmente debe realizarse drenaje del absceso u orquiectomía para curar la enfermedad $(5,7)$.

\section{R. López Rodríguez, S. Arias Rivas, E. González Babarro, C. Martínez Rey, M. R. Alende Sixto}

Unidad Médica de Alta Precoz. Servicio de Medicina Interna. Complejo Hospitalario Universitario. Santiago de Compostela. A Coruña

1. Young EJ. An overview of human brucellosis. Clin Infect Dis 1995; 21: 283-9.

2. Alapont JM, Gomez L, Delgado F, et el. Brucellar orchiepididymitis. Actas Urol Esp 2004; 28: 774-6.

3. Ibrahim AI, Awad R, Shetty SD, et al. Genito-urinary complications of brucellosis. Br J Urol 1988; 61: 294-8.

4. Afsar H, Baydar I, Sirmatel F. Epidídimo-orchitis due to brucellosis. Br J Urol 1993; 72: 104-5.

5. Navarro-Martínez A, Solera J, Corredoira J, et al. Epididymoorchitis due to Brucella melitensis: a retrospective study of 59 patients. Clin Infect Dis 2001; 33: 2017-22.

6. Papatsoris AG, Mpadra FA, Karamouzis MV, Frangides CY. Endemic brucellar epididymo-orchitis: a 10-year experience. Int J Infect Dis 2002; 6: 309-13.

7. González Sánchez FJ, Encinas Gaspar MB, Napal Lecumberri S, Rajab R. Brucellar orchiepididymitis with abscess. Arch Esp Urol 1997; 50: 289-92.

\section{Masa inguinal como forma de presentación de la enfermedad por arañazo de gato}

\section{Sr. Director:}

La enfermedad por arañazo de gato es producida en casi todos los casos por la Bartonella henselae como lo demuestran los diversos estudios inmunohistoquímicos y de cultivo en los ganglios de los pacientes afectados y en la sangre de los gatos transmisores así como en sus pulgas. Sin embargo, se ha especulado la posibilidad de que otros agentes como la Afipia felix o la Bartonella clarridgeiae puedan producir cuadros similares a esta enfermedad (1). La forma clínica típica de presentación es la aparición de una pápula o pústula cutánea tras contacto con el animal infectado, seguida a las pocas semanas de linfadenopatías ipsilaterales y malestar general con fiebre y sintomatología digestiva. Existen cuadro clínicos atípicos como el síndrome de Parinaud oculoglandular (conjuntivitis granulomatosa más linfadenitis preauricular ipsilateral), neumonitis atípica, osteitis y síndromes neurológicos (encefalopatía y neurorretinitis) $(1,2)$. Recientemente se han descrito, como casos muy infrecuentes, fiebre de origen desconocido en niños (3) y masas torácicas abcesificadas (4). El diagnóstico se basa además del cuadro clínico y del antecedente de contacto con gatos o perros, en el estudio histológico de las adenopatías con patrón de granulomas epiteloides con microabscesos y ocasional aparición de células de Langhans (1). Los test serológicos mediante inmunofluorescencia indirecta (IFI) son los más habituales en cuanto a confirmación diagnóstica. El pronóstico es bueno en general pues la sensibilidad a la mayoría de los antibióticos es alta y en sus formas típicas, la evolución natural es hacia la resolución espontánea (2).

Presentamos el caso de una mujer de 23 años que refería como único antecedente de interés la adquisición de un gato como mascota unos meses antes de la presentación de un bulto- 